\title{
DISTANCE AND HOPS-BASED ENERGY ESTIMATION IN WIRELESS SENSOR NETWORKS
}

\author{
PRADEEP KUMAR TS, SAYALI CHITNIS
}

Department of CS and SE, SCSE, VIT University, Chennai, Tamil Nadu, India. Email:asha.s@vit.ac.in

Received: 23 January 2017, Revised and Accepted: 03 March 2017

\begin{abstract}
The world of internet of things (IoT) and automation has been catching a robust pace to impact wide range of commercial and domestic applications for some time now. The IoT holds ad-hoc or wireless sensor networks (WSNs) at its very primary implementation level, the hardware level. The increasing requirement of these networks demands a renewed and better design of the network that improves the already existing setbacks of WSNs, which is mainly the power consumption and optimization. Routing highly affects the power consumed in the nodes in WSNs, hence having a modified routing algorithm which is specific to the application and meets its needs, particularly it is a good approach instead of having a generalized existent routing approach. Currently, for WSN having adequate number of nodes, routing involves maximum number of nodes and hops so as to reduce power consumption. However, for restricted areas and limited nodes, this scenario concludes with using up more number of nodes simultaneously resulting in reducing several batteries simultaneously. A routing algorithm is proposed in this paper for such applications that have a bounded region with limited resources. The work proposed in this paper is motivated from the routing algorithm positional attribute based next-hop determination approach (PANDA-TP) which proposes the increase in number of hops to reduce the requirement of transmission power. The aim of the proposed work is to compute the distance between the sending and receiving node and to measure the transmission power that would be required for a direct (path with minimum possible hops) and a multi-hop path. If the node is within the thresh-hold distance of the source, the packet is undoubtedly transferred directly; if the node is out of the thresh-hold distance, then the extra distance is calculated. Based on this, the power boosting factor for the source node, and if necessary, then the extra number of nodes that would be required to transmit is determined. An extra decision-making step is added to this approach which makes it convenient to utilize in varied situations. This routing approach suits the current level of robustness that the WSNs demand.

Keywords: Wireless networks, Sensors, Power estimation, Wireless sensor networks.
\end{abstract}

(c) 2017 The Authors. Published by Innovare Academic Sciences Pvt Ltd. This is an open access article under the CC BY license (http://creativecommons. org/licenses/by/4. 0/) DOI: http://dx.doi.org/10.22159/ajpcr.2017.v10s1.19571

\section{INTRODUCTION}

Both the commercial users and the research fields have shown an increased interest in the wireless sensor network (WSN) technology recently. WSN is a structured or unstructured arrangement of nodes (i.e., sensors motes or transducers) deployed in a geographical area for continuous monitoring and recording the activities of that area. It records whether any additions, eliminations, or variations are introduced in the area being monitored.

The area may be bounded or unbounded and the nodes can be mobile or immobile depending upon the application. Typically, a sensor mote is small device which has a sensing subsystem for data acquisition, processing subsystem for local data processing, and wireless data communication system [6].

WSNs are being deployed in a huge range of applications from health, space, and military to commercial and environmental observations and data analysis. However, due to its wireless nature, several constraints are imposed on the operation of the network. The nodes in the WSNs are self-powered, i.e., they work on batteries, which make it limited resource. The power is used in every other task such as computation, transmission continuous monitoring, and making power management a critical issue to be addressed in WSNs. There are performance factors that directly depend on power availability in the nodes. The node ability to transmit over long ranges, transmit for a longer time, and transmit with required signal strength to avoid noise interference depends on the power available in the battery of the node.

The network link lifetime is the time till the last node drains out of all of its energy and is expected to extend till the required application request is fulfilled. Along with these, the link quality, channel bandwidth, throughput, average end-to-end time delay, and average residual energy are some other factors that have to be optimized as per the demands of the applications. The designing of routing protocols thus takes care of all or few of the network optimization parameters. The routing in turn depends on the application requirements, whether it is over a bounded or unbounded area, whether it is time critical or data critical, etc. The routing algorithm should be given the decision-making power on that level where it decides to use which particular technique is to be used for building a route depending on the existing configuration and network conditions at that moment.

The objective of the paper is to develop an algorithm that analyzes the network requirements and capabilities at the needed time and make a decision to build a route either involving minimum number of nodes, while transmitting data to a destination residing outside the range cover of the source node, and reduce the total transmission power required or uses maximum hops route if it is affordable in that area of the network. In the former, the nodes evicted from the route save their energy, however, at the cost of extra boosted transmission energy from the source.

The WSN considered for experimental purpose is the one having a fixed configuration over a bounded area. The nodes in the WSN are immobile though the routing algorithm can be conveniently extended to immobile networks as well. These days, it can be easily made possible since global positioning system (GPS) technology is cheaper and produces adequately accurate results. Li and Mohapatra in their work have proposed certain localization techniques [2].

A good amount of work has been done already in the power optimization domain of WSN. The routing protocols can be proactive (e.g., differential source distance vector) or reactive (e.g., dynamic source routing [DSR], Ad-hoc on-demand distance vector [AODV], temporally ordered routing algorithm). The proactive protocols 
maintain the complete network routing information in every node at all times by periodically updating the location information and depend on the periodic updating of routing information. However, in this paper, reactive protocols (AODV) are utilized for simulation and performance measurement of the proposed HEAD route discovery protocol. In on-demand protocols, the route is created between the sender and receiver only when it is requested by the sender based on flooding mechanism.

Location-aided routing [5] protocol is an improvement over DSR protocol. It restricts the flooding region using the relative geographical information of the nodes. It makes use of the geographical information to decide the direction, in which the flooding should be propagated. If the possibility of availability of a node in a direction is absolutely zero, then the routing packets are dropped by the intermediate nodes and not flooded further. This was an effective step toward fighting against the network jamming and congestion.

Shelby et al. proposed that for an embedded wireless network, the data transfers always need not be through multihop forwarding strategy, but sometimes a direct transfer to the sink, i.e., the destination node has better advantages and does not affect the network lifetime.

Kee-Young et al. proposed a reliable energy aware routing [3] protocol, which extends the network lifetime by considering the residual energy left with the nodes after implementing the route. This energy is next time taken into consideration before reusing the same node for transmission. The success of data packet transmission is informed by enabling the transmission of data acknowledgment (DATA_ACK) packets to the concerned upstream node till the sender node.

Ganesh and Amrutha [8] suggested an improvement in the existing transmission control/internet protocol (TCP/IP). These are generally not used in wireless environment owing to their poor performance. However, this paper proposed the implementation of TCP/IP segment caching which improved the network performance considerably. The complete stream of data is expected to be broken down into segments and stored up or more appropriately cached before transmitting further by the individual nodes.

Barfunga et al. [7] proposed a cluster-based hierarchical protocol which showed comparatively improved results, under specific assumptions, over the low-energy adaptive clustering hierarchy protocol. In this, the base station selects the cluster-heads among the available nodes in the network, based on several factors such as the distance of the node from the base station and its residual energy. This protocol is based on time division multiplexing access concepts and works in the sublevel of the data-link layer, MAC layer.

Kumar and Thomas [10] recently suggested a maximum amount shortest path protocol that utilized the ant colony optimization as its solution. It focuses more on maximum data collection by the sink and residual energy in the nodes. The problem formulated was in terms of mathematical function that used linear integer programming. The region is divided into partitions based on zones. However, in this routing technique, the mobility of nodes is constrained along restricted path for getting improved efficiency results. The optimal route is built by initially calculating the residual energy in every node and then by taking into account the channel noise, delay, shortest distance to the destination node, and residual energy.

Li and Mohapatra put forth a positional attribute-based algorithm, PANDA protocol where based on the relative location of a node; it decides whether it is a good or bad candidate for forwarding a particular packet. This way the excessive flooding of control packets is reduced. This algorithm is further divided into sub-algorithms based on three cases:
1. PANDA LO

2. PANDA-TP

3. PANDA-LV.

The algorithm is based on the fact that for a transmission, if the number of sequential hops is increased, both the rebroadcast delay and the total transmission power will be reduced. The range of transmission in this protocol is aimed to be kept as low as possible. However, this sets the condition on the network to necessarily be populated with more number of nodes or at least adequate number of nodes and effectively reduce the power consumed per node owing to the reduced range of transmission. Here also, the use of GPS is made for gathering the location information to restrict the flooding of control packets while setting up the route.

Tan et al. proposed a PMAR protocol, power and mobility aware routing, a heuristic scheme that focuses on maximizing the minimum residual battery level and minimizing the total transmission power. It also shows improvement in the total end-to-end delay as compared to hierarchical energy-aware protocol and PANDA protocols. This protocol however does not present any implementation conclusions, being a heuristic scheme.

\section{PROBLEM STATEMENT}

Based on the assumptions made, the following problem statement is made for the work proposed: For every source node belonging to immobile WSN for transmitting data to a destination node residing outside its maximum threshold distance, among all possible routes between the two nodes, the route selected must involve minimum number of nodes or hops, only if it consumes lesser total average energy after boosting the transmission power of the source node. Here, the total average transmission power becomes the decision point for choosing the routing building technique.

Mathematically,

$\mathrm{m} \in\left\{\mathrm{Ls} \mid \mathrm{P}_{\mathrm{T}}^{\mathrm{m}}=\min \left(\mathrm{P}_{\mathrm{T}(\text { direct })}, \mathrm{P}_{\mathrm{T}(\text { Indirect })}\right)\right\}$

The symbols and variables used are explained in Table 1.

The algorithm for choosing a path under the assumed conditions: (1) the nodes of the WSN are stationary in time and (2) the given network consists of adequate number of nodes depending on the application.

Table 1: Symbols and description in the algorithm

\begin{tabular}{|c|c|}
\hline Symbol & Meaning \\
\hline $\mathrm{D}_{\text {Total }}$ & The total distance between source and destination nodes \\
\hline $\mathrm{D}_{\mathrm{Th}}^{\text {lotal }}$ & The threshold distance \\
\hline $\mathrm{P}_{\mathrm{T} \text { (Direct) }}$ & $\begin{array}{l}\text { The transmission power required when transmitting } \\
\text { directly to destination node with minimum } \\
\text { possible hops }\end{array}$ \\
\hline $\mathrm{P}_{\mathrm{T} \text { (Indirect) }}$ & $\begin{array}{l}\text { The transmission power when transmitting to the } \\
\text { destination via multiple hops }\end{array}$ \\
\hline$P_{s-d}$ & $\begin{array}{l}\text { Transmission power required while transmitting directly } \\
\text { from source to destination }\end{array}$ \\
\hline M & Total number of routes between source and destination \\
\hline $\mathrm{m}$ & Route number \\
\hline $\mathrm{D}_{\text {imjm }}$ & Distance on the $\mathrm{m}^{\text {th }}$ route between node $\mathrm{i}$ and $\mathrm{j}$ \\
\hline $\mathrm{H}^{\mathrm{imj}}$ & Total number of hops \\
\hline $\mathrm{T}_{\mathrm{k}}^{\mathrm{m}}$ & $\begin{array}{l}\text { Total number of paths having } \mathrm{k} \text { hops between source and } \\
\text { destination }\end{array}$ \\
\hline $\mathrm{P}_{\mathrm{T}}^{\mathrm{m}}$ & Total transmission power along $\mathrm{m}^{\text {th }}$ route \\
\hline $\mathrm{D}_{\text {Thi }}$ & Total threshold distance \\
\hline Ls & Set of total number of paths \\
\hline $\mathrm{P}_{\text {Timjm }}$ & $\begin{array}{l}\text { Total transmission power from } \mathrm{i}^{\text {th }} \text { to } \mathrm{j}^{\text {th }} \text { node on } \\
\mathrm{m}^{\text {th }} \text { route }\end{array}$ \\
\hline
\end{tabular}




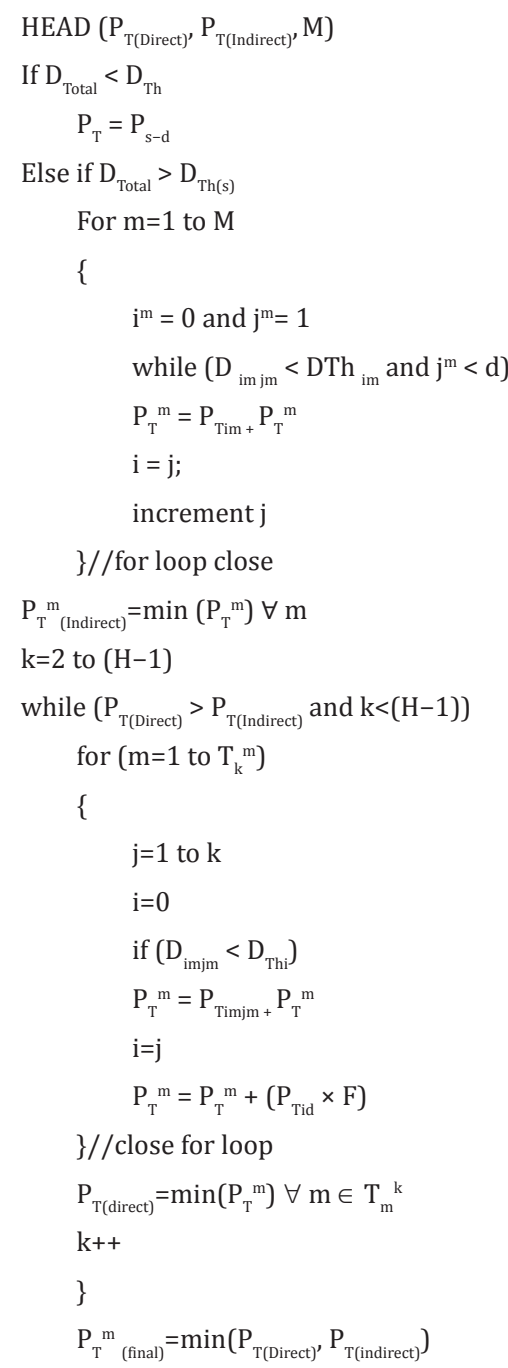

The HEAD algorithm requires the direct transmission power and the indirect transmission power that the one which would make use of maximum number of nodes and hops to reach the destination that is calculated and that has to be compared and further route building has to be initiated which is described further (Table 1).

The algorithm shows that a trade-off between the boosting factor and extra number of hops is made to determine a suitable path between the source and destination node anytime.

Initially, all the possible paths between source and destination are determined. It is an easy operation due to availability of location information of a node and stationary nature of the network.

Now depending on the location of the destination node, if it is presentwithin the threshold distance of the source node, then a direct transmission from source to destination node is performed. If the destination node is present outside the threshold distance of the source node, the excessive distance outside the range is calculated, based on which the boosting factor of the transmission power will be determined. Now, the transmission power for direct transmission $\left(\mathrm{P}_{\mathrm{t}}\right)$ required is calculated having minimum possible hops. Furthermore, the corresponding boosting factor for the intermediate nodes is calculated from which there is a probability of direct transmission further. The transmission power involving the necessary number of hops which eliminates the boosting of any (source or intermediate) node, i.e., the indirect transmission power (Pt-indirect) is calculated with the initial step.

At every possible hop count from $1 \ldots \mathrm{n}-1$, where $\mathrm{n}$ is the number of nodes from sender to receiver, the algorithm compares whether at that stage after boosting the transmission power of the intermediate node and whether the direct transmission power is less than the indirect transmission power. As soon as this condition bears true, this particular intermediate node is used for direct packet transmission to the destination. If this condition does not hold true till the $\mathrm{n}^{\text {th }}$ node, then by default the indirect transmission route is chosen for data transmission. This way at every step the probability of optimizing the power while routing is kept in check.

The focus of the proposed work is also over determining the optimal number of nodes that need to be disturbed for a transmission, such that they do not affect the lifetime of a network due to extra usage of power from less number of nodes. Hence, there is a trade-off or compromise to be experienced between the hops that need to be encountered and the power boosting factor for source node. The power boosting factor if shows a higher risk as compared to opting the greater number of hops, then the latter is chosen for route generation.

\section{EXPERIMENTAL RESULTS}

The algorithm mentioned in section 2 is tested for WSNs using the simulator ns2. For generating and simulating the shown WSN network, network simulator and network animator are used. These two tools are very popularly used for the designing, simulation, and analysis of any network. The tool generates a trace file that records all the events that are happening during the transmission and other events. The trace file is used for analysis purpose and keeps the records of activity in the network for every second. This resolution is modifiable and can be increased up to nanoseconds by adjusting the logic in the network simulator code. The network generated is simulated by setting certain parameters as shown in Fig. 1. The analysis of trace file is yet to be included in the research work proposed.

Table 2 shows the parameters that are set for the distance and hopsbased algorithm. Setting of these values is shown in Fig. 1.

Fig. 2 represents an immobile WSN deployed over a bounded region. It has 13 nodes which suggest that for the bounded region, only adequate number of nodes is present.

Fig. 3 shows a simulated network in network animator. It is configured as a duplex network. TDP-IP as well as UDP protocol is applied for data transfer between the nodes to test performance in both cases.

Shown are the output screenshots of simulating certain scenarios where the proposed work can be explained. As we can see in the shown example, there are total 13 nodes in the network. Node $0,3,7$ are source

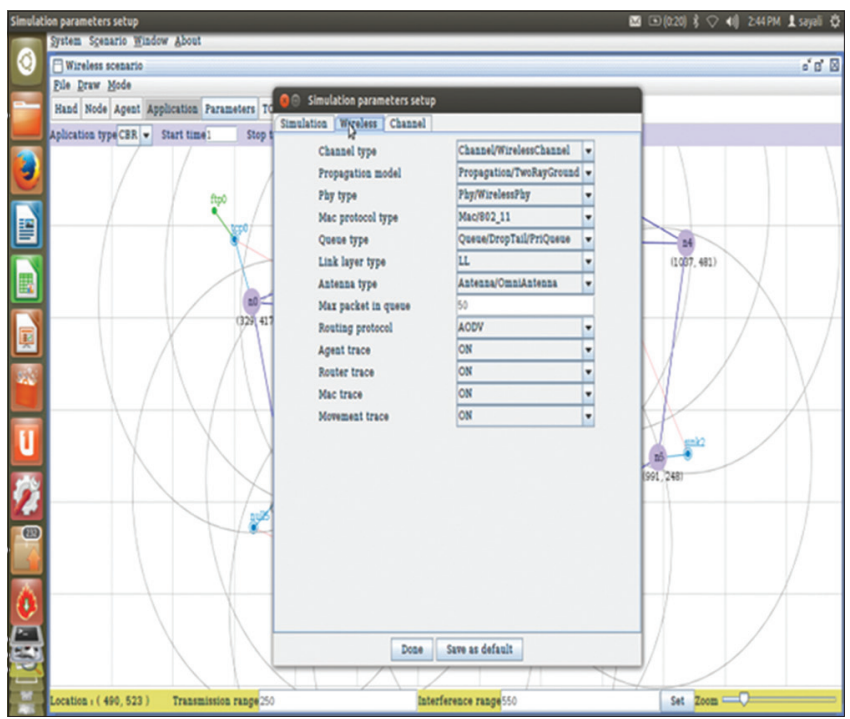

Fig. 1: Parameters set for wireless sensor networks 


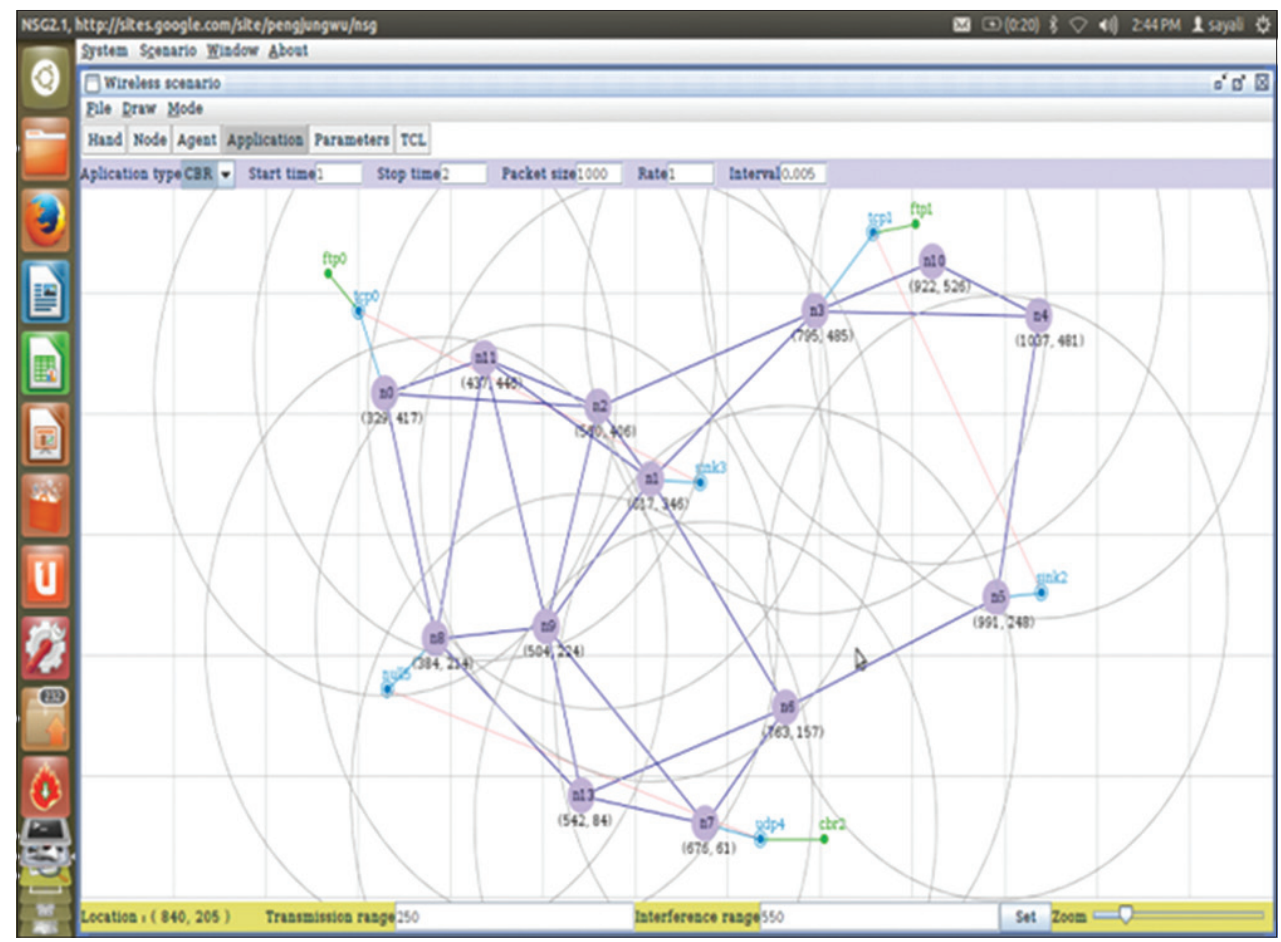

Fig. 2: Sample wireless sensor networks using NSG2.1

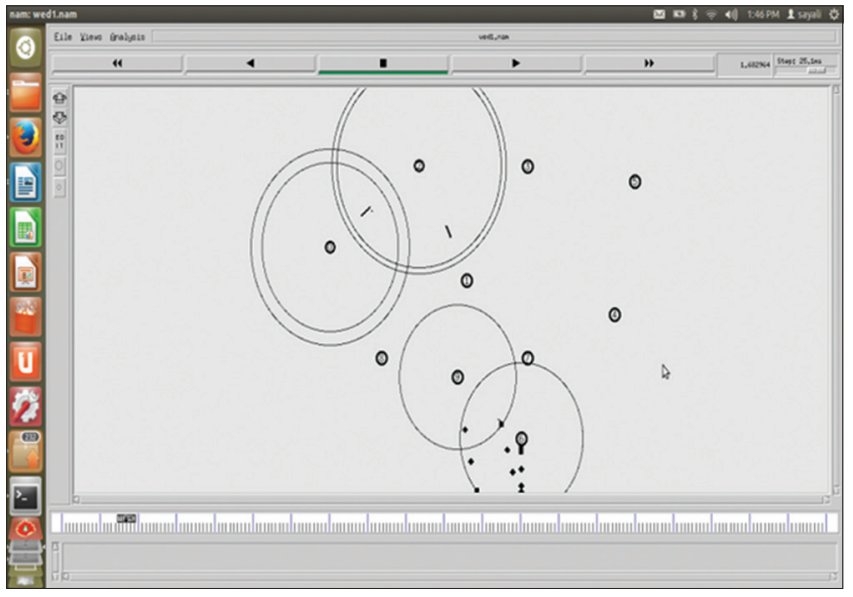

Fig. 3: Simulation of wireless sensor networks in Nam

Table 2: Parameters set for algorithm

\begin{tabular}{ll}
\hline Metrics & Value \\
\hline Protocol & AODV, DSR, and distance/hop Algorithm \\
LL & Link layer (default) \\
MAC & $\begin{array}{l}\text { IEEE } 802.11 \text { is used rather IEEE 802.15.4 (as we } \\
\text { need huge data rate) }\end{array}$ \\
Channel & Wireless channel with ideal gain \\
Power & No power and energy specified (we have taken \\
default) & $1000 \times 1000 \mathrm{~m}$ \\
Workspace & 50 bytes \\
Queue size & Omni directional antenna \\
Antenna & ON for mobility, routing and agent \\
Traces & TFTP and FTP traffic \\
Application layer & \\
protocol & 13 nodes \\
Number of nodes &
\end{tabular}

AODV: Ad-hoc on-demand distance vector, DSR: Dynamic source routing nodes simultaneously. We consider the case of node 0 (n0) as the source node trying to transmit to destination node 1 ( 1 1). The node $n 1$ resides outside the range of $n 0$; hence, it has to reach $n 1$ through few hops necessarily. For this, it can prefer two paths which are either via n11 and $n 2$ or directly via $n 2$ only. Reducing the hop distance will reduce the required transmission power is the case proposed in PANDA-TP [1] protocol.

Fig. 4 shows the packet delivery ratio, one of the important results during the mobility of the node. The AODV and DSR protocol, the packet drop, is nominal from the beginning irrespective of the time they were not in mobility. However, our algorithm improves as the mobility decreases. This metric is important in terms of understanding the algorithm in a better way. Fig. 5 shows the throughput of the three protocols; the throughput of our algorithm outperforms well compared to the DSR and AODV protocol because the distance and hop, both are taken into consideration while the simulation time is increasing.

Here, it is not known initially whether the PANDA or HEAD algorithm provides better power optimization. Hence, the total average power required for indirect transmission, i.e., via n0-n11-n2-n1 is computed. Now, the power required for transmission via n0-n2-n1 is calculated. If this is comparatively low, then the power boost required for the source node for direct transmission to $\mathrm{n} 2$ is calculated further. Boosting the transmission power of the source node is hence performed here but with the amount that proves lesser than the total average power that would be required to transmit using maximum nodes and hops. This not only results in depleting less number of batteries at a time, but also since only minimum nodes are used, the delay in the transmission is also reduced. The possibility of data tampering and security threats is indirectly reduced since the chance of manipulating a node for misbehavior reduces with less number of nodes involved in the whole transmission.

\section{CONCLUSION}

In this paper, a novel algorithm, HEAD, that takes the routing to a smarter level by utilizing its decision-making ability to develop 


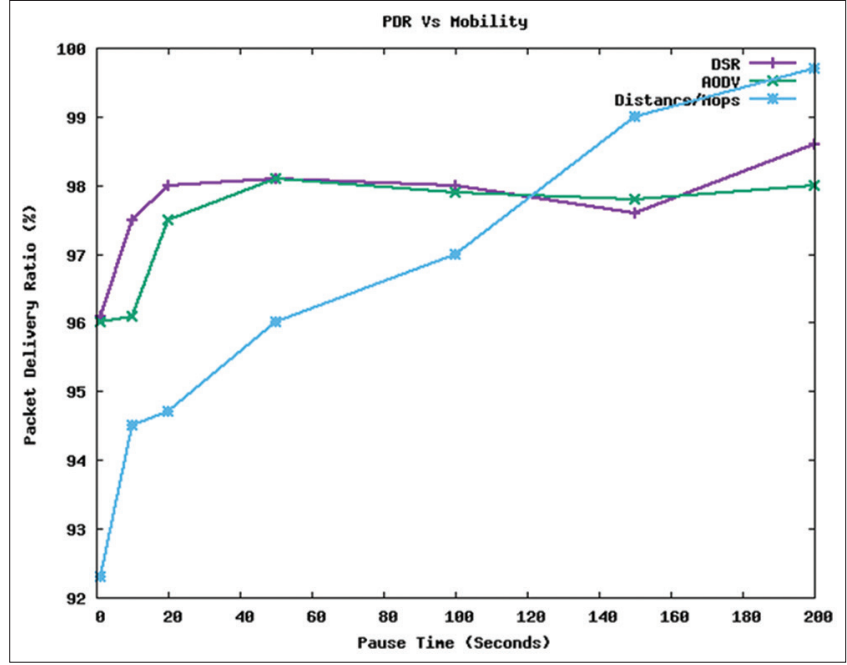

Fig. 4: Packet delivery ratio

routes on demand is developed and verified successfully on simulated environments. NS2, Nam, and Network simulator graph were used for the same purpose. The algorithm shows a fail-safe way to design routes for WSNs, thus improving the network efficiency and lifetime. The decision taken is supported by a series of computations done on the source node before the transmission begins.

\section{REFERENCES}

1. Li J, Mohapatra P. PANDA: An Approach to Improve Flooding Based Route Discovery in Mobile Ad hoc Networks. Technical Report CSE-2002-29. Davis: University of California; 2002.

2. Tan WC, Bose SK, Cheng TH. Power and mobility aware routing in wireless ad hoc networks. IET Commun 2012;6(11):1425-37.

3. Shin KY, Song J, Kim J, Yu M, Mah PS. REAR: Reliable energy aware routing protocol for wireless sensor networks. In: The $9^{\text {th }}$ International Conference on Advanced Communication Technology. IEEE. Vol. 1. February 12, 2007. p. 525-3.

4. Shelby Z, Pomalaza-Raez C, Karvonen H, Haapola J. Energy optimization in multihop wireless embedded and sensor networks. Int $\mathbf{J}$

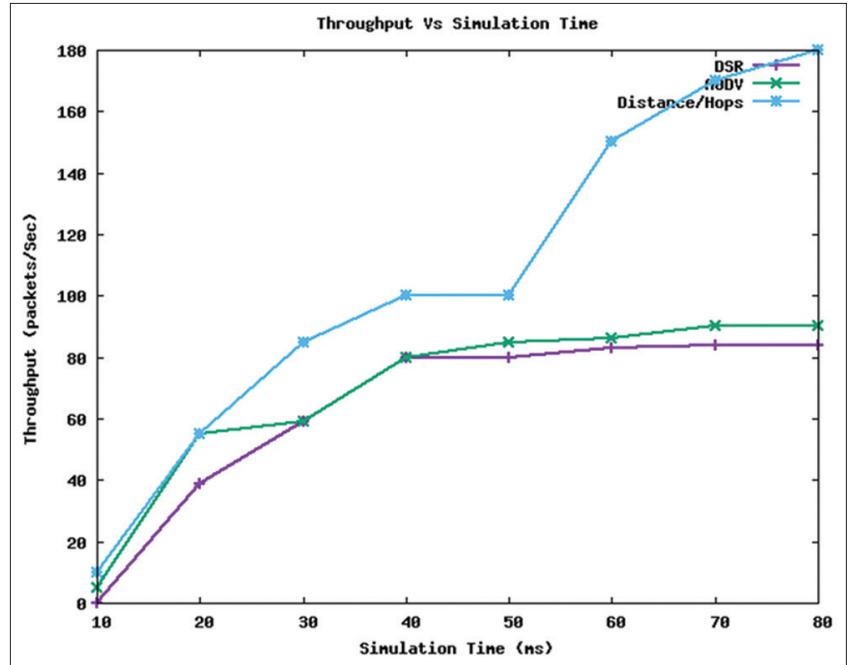

Fig. 5: Throughput

Wirel Inf Netw 2005;12(1):11-21.

5. Ko YB, Vaidya NH. Location-aided routing (LAR) in mobile ad hoc networks. Wirel Netw 2000;6(4):307-21.

6. Anastasi G, Conti M, Di Francesco M, Passarella A. Energy conservation in wireless sensor networks: A survey. Ad Hoc Netw 2009;7(3):537-68.

7. Barfunga SP, Rai P, Sarma HK. Energy efficient cluster based routing protocol for Wireless Sensor Networks. In: Computer and Communication Engineering (ICCCE), International Conference on 2012 July, 3. IEEE; 2012. p. 603-7.

8. Ganesh S, Amutha R. Energy efficient transport protocol for wireless sensor networks. In: Computer Science and Information Technology, ICCSIT 2009. $2^{\text {nd }}$ IEEE International Conference on 2009 August, 8. IEEE; 2009. p. 464-8.

9. Beyme S, Leung C. A stochastic process model of the hop count distribution in wireless sensor networks. Ad Hoc Netw 2014;17:60-70.

10. Kumar NA, Thomas A. Energy efficiency and network lifetime maximization in wireless sensor networks using improved ant colony optimization. In: Computing Communication \& Networking Technologies (ICCCNT), 2012 Third International Conference on 2012 July, 26 IEEE. p. 1-5 